\title{
Calibration of Smartphone Light Sensors with a Near Field Communication Enabled Reference
}

\author{
Tore Leikanger, Christian Schuss and Juha Häkkinen \\ Circuits and Systems (CAS) \\ University of Oulu \\ Oulu, Finland \\ Email: firstname.lastname@ee.oulu.fi
}

\begin{abstract}
This paper describes a technique for calibrating the internal ambient light sensor (ALS) in smart phones. The calibration process depends on Near-Field Communication (NFC). The system consists of an NFC core implemented in an Field-Programmable Gate-Array (FPGA) development platform featuring the NFC analog front end circuit. In the proposed system, the smart phone interacts directly with a reference ALS through the NFC device The direct communication is possible with the help of a custom NFC command that controls an InterIntegrated Circuit (I2C) master core in the FPGA. After the calibration process of the ALS in a smart phone is completed, the accuracy of the measurement is greatly improved. This is helpful to obtain precise information on the light intensity in indoor surroundings.
\end{abstract}

Keywords-ambient light sensor; calibration; smartphone; nearfield communication; NFC; inter-integrated circuit; I2C

\section{INTRODUCTION}

Nowadays, the majority of smart phones come with an builtin ALS, usually used for adjusting the brightness of the screen. Other uses have also been proposed earlier, e.g. to use the internal light sensor to calculate the amount of energy solar chargers can give in different environments. [1].

However, the problem with the internal light sensor is the large uncertainty in the ambient light measurements [2]. The differences in the measured ambient light intensities $\left(E_{v}\right)$ are not only due to different smart phone models, also large differences between the same smart phone models have been shown [3].

In this paper, we present a system for calibrating the ambient light sensors in near field communication (NFC) enabled smart phones. This system is based on an NFC to Inter-Integrated Circuits (I2C) interface device, which we have developed [4], and is connected with a reference ambient light sensor. The NFC-to-I2C interface enables smart phones featuring NFC to interact directly with the $\mathrm{I} 2 \mathrm{C}$ enabled circuit, in this case with an ambient light sensor. The interaction works through a custom NFC request, which controls the I2C master core in the NFC-to-I2C interface, making the smart phone the master on the $\mathrm{I} 2 \mathrm{C}$ bus [4].

NFC is used as the interface between the smart phone and the reference ambient light sensor, in order to achieve a short distance between the smart phone sensor and the reference sensor [5]. A short distance is needed for the reference sensor so that the same ambient light intensity is measured as with the internal sensor in the smart phone.

This paper is divided into three sections. Section II introduces the system, the measurement data is presented in section III, and discussed in section IV.

\section{MEASUREMENT SYSTEM}

The measurement system is based on an NFC enabled fieldprogrammable gate-array (FPGA) device as the NFC-to-I2C interface [4]. The NFC-to-I2C interface is connected to the TSL2561 ambient light sensor by TAOS (Texas Advanced Optoelectronic Solutions) [6]. We have evaluated this sensor and compared the results with commercial light measurement tools, a luxmeter, as well as smart phone measurements. This evaluation shows that the TSL2561 sensor is reliable in indoor environments, and can be used as reference for calibration of the internal ambient light sensors in smart phones. [3]

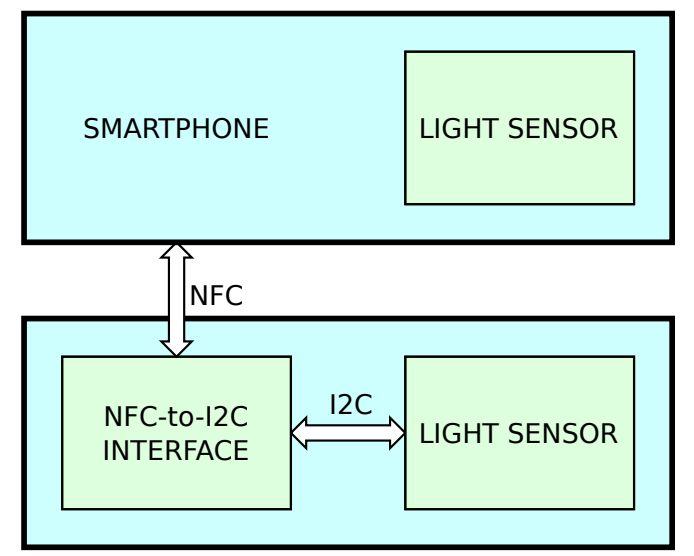

Fig. 1. Block diagram of the measurement system.

Fig. 1 shown the block diagram of the measurement system set-up, and a picture of the system can be seen in Fig. 2. In measurements, the smart phone measures the ambient light using the internal ALS at the same time as the external reference ALS. The measurement data of the external sensor is then fitted as a function of the measurement data of the internal sensor. After the data is fitted, the actual light intensity can be calculated using the equation acquired in the process. For this approach to work, we need the sensor data to be measured 


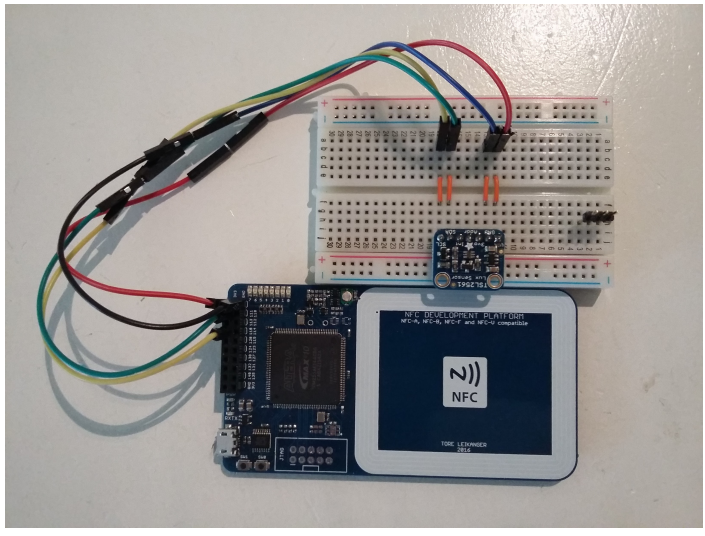

Fig. 2. Picture of the measurement system. The NFC-to-I2C interface is powered through a USB cable not shown in the picture.

in sufficiently many light intensities, and preferably over a large spectre. We controlled the light intensity by changing the distance between the lamp and the measurement system.

A second order polynomial is fitted to the measurement using the least squares method. In order to fit a second order polynomial

$$
p(x)=b_{0}+b_{1} x+b_{2} x^{2}
$$

to the measurement data, the coefficients $b_{0}, b_{1}$ and $b_{2}$ must satisfy the equations [7]

$$
\begin{aligned}
b_{0} n+b_{1} \sum x_{i}+b_{2} \sum x_{i}^{2} & =\sum y_{i}, \\
b_{0} \sum x_{i}+b_{1} \sum x_{i}^{2}+b_{2} \sum x_{i} 3 & =\sum x_{i} y_{i}, \\
b_{0} \sum x_{i}^{2}+b_{1} \sum x_{i}^{3}+b_{2} \sum x_{i}^{4} & =\sum x_{i}^{2} y_{i} .
\end{aligned}
$$

We solved these equations for the coefficients using MATLAB. By using the notation

$$
\begin{aligned}
\sum x_{i} & =S_{x}, \quad \sum x_{i}^{2}=S_{x^{2}}, \quad \sum x_{i}^{3}=S_{x^{3}}, \\
\sum x_{i}^{4} & =S_{x^{4}}, \quad \sum x_{i} y_{i}=S_{x y}, \quad \sum x_{i}^{2} y_{i}=S_{x^{2} y},
\end{aligned}
$$

we obtained

$$
\begin{array}{r}
b_{0}=\frac{S_{x y}\left(S_{x} S_{x^{4}}-S_{x^{2}} S_{s^{3}}\right)-S_{x^{2} y}\left(S_{x^{2}}^{2}+S_{x} S_{x^{3}}\right)}{S_{x^{4}} S_{x}^{2}-2 S_{x} S_{x^{2}} S_{x^{3}}+S_{x^{2}}^{3}-n\left(S_{x^{4}} S_{x^{2}}+S_{x^{3}}^{2}\right)} \\
-\frac{S_{y}\left(-S_{x^{3}}^{2}+S_{x^{2}} S_{x^{4}}\right)}{S_{x^{4}} S_{x}^{2}-2 S_{x} S_{x^{2}} S_{x^{3}}+S_{x^{2}}^{3}-n\left(S_{x^{4}} S_{x^{2}}+S_{x^{3}}^{2}\right)}, \\
b_{1}=\frac{S_{t} y\left(S_{x} S_{x^{4}}-S_{x^{2}} S_{x^{3}}\right)-S_{x^{2} y}\left(S_{x} S_{x^{2}}-n S_{x^{3}}\right)}{S_{x^{4}} S_{x}^{2}-2 S_{x} S_{x^{2}} S_{x^{3}}+S_{x^{2}}^{3}-n\left(S_{x^{4}} S_{x^{2}}+S_{x^{3}}^{2}\right)} \\
-\frac{S_{x y}\left(-S_{x^{2}}^{2}+n S_{x^{4}}\right.}{S_{x^{4}} S_{x}^{2}-2 S_{x} S_{x^{2}} S_{x^{3}}+S_{x^{2}}^{3}-n\left(S_{x^{4}} S_{x^{2}}+S_{x^{3}}^{2}\right)},
\end{array}
$$

$$
\begin{aligned}
b_{2} & =\frac{S_{y}\left(S_{x^{2}}^{2}-S_{x} S_{x^{3}}\right)-S_{x y}\left(S_{x} S_{x^{2}}-n S_{x^{3}}\right)}{S_{x^{4}} S_{x}^{2}-2 S_{x} S_{x^{2}} S_{x^{3}}+S_{x^{2}}^{3}-n\left(S_{x^{4}} S_{x^{2}}+S_{x^{3}}^{2}\right)} \\
& +\frac{S_{x^{2} y}\left(S_{x}^{2}-n S_{x^{2}}\right)}{S_{x^{4}} S_{x}^{2}-2 S_{x} S_{x^{2}} S_{x^{3}}+S_{x^{2}}^{3}-n\left(S_{x^{4}} S_{x^{2}}+S_{x^{3}}^{2}\right)}
\end{aligned}
$$

We developed an Android application to perform these measurements, and to find the correlation between the internal and the reference sensor. The corrected value of the light intensity is presented, together with the light intensity as measured by both the internal sensor and the reference sensor respectively. A screen-shot of this Android application can be seen in Fig. 3.

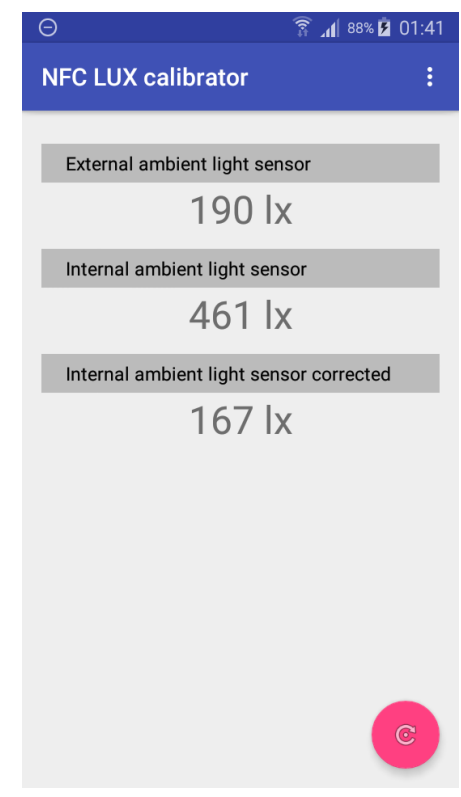

Fig. 3. Screen-shot of the Android application.

\section{MEASUREMENT DATA}

Table I presents measurement data obtained with the internal sensor of the smart phone and the reference sensor. In addition, the difference in absolute readings $\left(E_{v, \text { int }}-E_{v, \text { ref }}\right)$ is shown. The data are selected randomly throughout the measurement range. Further, we have calculated a second order least squares regression curve (equations (1) to (7)) to find the correlation between the internal and reference sensors. We found the regression curve to follow the equation

$$
\begin{aligned}
E_{v, c a l} & =b_{0} E_{v}^{2}+b_{1} E_{v}+b_{2} \\
& =-9.73 \times 10^{-6} E_{v}^{2}+0.36 E_{v}+31.35
\end{aligned}
$$

where $E_{v}$ is the measured ambient light intensity and $E_{v, c a l}$ is the calibrated ambient light intensity.

The regression curve is plotted together with the measurement data and the error of the regression curve in Fig. 4. The corrected values of the measurement data in Table I are also shown in Table II. 


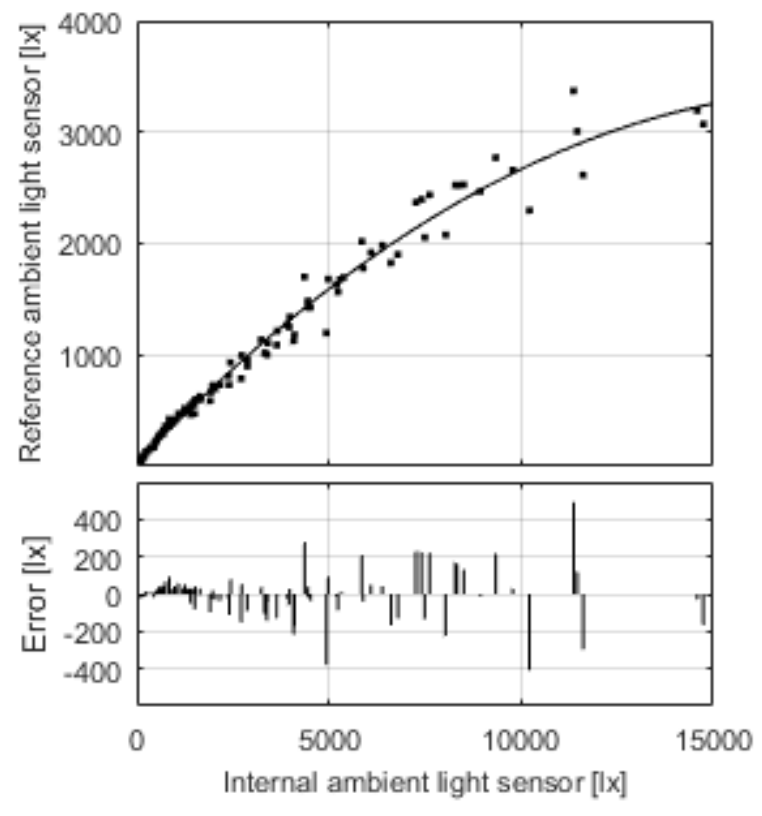

Fig. 4. Plot of the reference sensor data against the internal sensor data under an halogen lamp as black dots. A second order polynomial least squares fit is plotted as a solid line. The lower plot shows the difference between the measurement data and the fit

TABLE I

SELECTED MEASUREMENT DATA WHEN USING AN HALOGEN LIGHT BULb.

\begin{tabular}{cccc}
\hline \multicolumn{2}{c}{ Sensor readings [1x] } & \multicolumn{2}{c}{ Difference } \\
Internal sensor & Reference sensor & Absolute [1x] & Relative [\%] \\
\hline 101 & 51 & +50 & +98 \\
208 & 91 & +117 & +129 \\
505 & 229 & +276 & +121 \\
1001 & 418 & +583 & +139 \\
1499 & 550 & +949 & +173 \\
2018 & 696 & +1322 & +190 \\
3238 & 1135 & +2103 & +185 \\
4982 & 1680 & +3302 & +197 \\
7265 & 2369 & +4896 & +207 \\
8940 & 2468 & +6472 & +262 \\
\hline
\end{tabular}

\section{RESULTS}

The measurement data shows that it is possible to calibrate the sensor using the NFC enabled ambient light sensor device. As can be seen in Tables I and II, the absolute difference between the corrected data and the reference regression curve seems to increase with the light intensity. However, the relative difference does not increase as rapidly. The calibrated data has a standard deviation $\sigma=115.9 \mathrm{~lx}$ from the reference when data over the whole measurement range is taken into consideration. The calibration of the light sensor using this technique reduces the difference, from being at maximum more than $250 \%$ before calibration to less than $35 \%$ after calibration. This means that calculations done using the internal
TABLE II

SELECTED CORRECTED MEASUREMENT DATA WHEN USING AN HALOGEN LIGHT BULB.

\begin{tabular}{cccc}
\hline \multicolumn{2}{c}{ Sensor readings [1x] } & \multicolumn{2}{c}{ Difference } \\
Internal sensor & Reference sensor & Absolute [1x] & Relative [\%] \\
\hline 68 & 51 & +17 & +33 \\
106 & 91 & +15 & +16 \\
211 & 229 & -18 & -8 \\
383 & 418 & -35 & -8 \\
550 & 550 & 0 & 0 \\
727 & 696 & +31 & +4 \\
1098 & 1135 & -37 & -3 \\
1588 & 1680 & -92 & -5 \\
2140 & 2369 & -229 & -10 \\
2480 & 2468 & +12 & +0 \\
\hline
\end{tabular}

ambient light sensor in a smart phone are much more precise if a calibration of the sensor was carried out. For example, when calculating the actual energy of solar chargers for smart phones, as proposed by C. Schuss et.al [1]. However, since solar cells are highly dependent on the light spectrum [1], the internal light sensor might have to be calibrated specifically for each kind of light source.

\section{CONCLUSION}

We have seen that the calibration of the internal ambient light sensor of a smart phone is possible using a simple second order regression. The measurement data in an uncalibrated sensor of a smart phone was overshooting the reference by up to more than $250 \%$. When the same sensor was calibrated, the average measured light intensity was close to the reference, with measurements deviating by less than $35 \%$. In other words, the precision of the sensor has increased significantly after the proposed calibration method. When using the calibration process to calculate the energy of solar chargers for smart phones, a separate calibration for different light sources might be necessary. The separate calibrations are necessary due to the different light spectra of light sources.

\section{REFERENCES}

[1] C. Schuss et al., Efficient use of solar chargers with the help of ambient light sensors on smartphones, Open Innovations Association (FRUCT16), 2014 16th Conf. of, Oulu, 2014, pp. 79-85.

[2] C. Schuss et al., Evaluating ambient conditions for solar chargers with the help of sensors on smartphones, 2015 IEEE Int. Instrumentation and Measurement Technology Conf. (I2MTC) Proc., Pisa, 2015, pp. 103-108.

[3] C. Schuss et al., Using Ambient Light Sensors in Smartphones for Evaluating Indoor Lighting Conditions, 14th IMEKO TC10 Workshop on Technical Diagnostics, Milan, 2016, pp. 258-263.

[4] T. Leikanger et al., Interfacing External Sensors with Android Smartphones Through Near Field Communication, 14th IMEKO TC10 Workshop on Technical Diagnostics, Milan, 2016, pp. 164-169.

[5] V. Coskun et al., A Survey on Near Field Communication (NFC) Technology, Wireless Personal Communications, 2013, vol. 71, num. 3, pp. 2259-2294.

[6] TSL2560, TSL2561 Light-to-digital converter, datasheet, 2009.

[7] E. Kreyszig, "Linear Numeric Algebra" in Advanced Engineering Mathematics, 10th ed. Ohio: Wiley, 2011, ch. 20, sec. 5, pp. 872-875. 